\title{
The convalescent sera option for containing COVID-19
}

\author{
Arturo Casadevall' and Liise-anne Pirofski ${ }^{2}$ \\ 'Department of Molecular Microbiology and Immunology, Johns Hopkins School of Public Health, Baltimore, Maryland, USA. ${ }^{2}$ Division of Infectious Diseases, Department of Medicine, Albert Einstein College \\ of Medicine, Bronx, New York, USA.
}

A s of early 2020, humanity is confronting a pandemic in severe acute respiratory syndrome coronavirus 2 (SARS-CoV-2). SARS-CoV-2 causes coronavirus disease, abbreviated as COVID-19. At the time of this writing, SARS-CoV-2 is spreading in multiple countries, threatening a pandemic that will affect billions of people. This virus appears to be a new human pathogen. Currently there are no vaccines, monoclonal antibodies (mAbs), or drugs available for SARS-CoV-2, although many are in rapid development and some may be available in a short time. This Viewpoint argues that human convalescent serum is an option for prevention and treatment of COVID-19 disease that could be rapidly available when there are sufficient numbers of people who have recovered and can donate immunoglobulin-containing serum.

\section{Passive antibody therapy}

Passive antibody therapy involves the administration of antibodies against a given agent to a susceptible individual for the purpose of preventing or treating an infectious disease due to that agent. In contrast, active vaccination requires the induction of an immune response that takes time to develop and varies depending on the vaccine recipient. Thus, passive antibody administration is the only means of providing immediate immunity to susceptible persons. Passive antibody therapy has a storied history going back to the 1890s and was the only means of treating certain infectious diseases prior to the development of antimicrobial therapy in the 1940s (1,2). Experience from prior outbreaks with other coronaviruses, such as SARS-CoV-1, shows that such convalescent sera contain neutralizing antibodies to the relevant virus (3).

In the case of SARS-CoV-2, the anticipated mechanism of action by which passive antibody therapy would mediate pro- tection is viral neutralization. However, other mechanisms may be possible, such as antibody-dependent cellular cytotoxicity and/or phagocytosis. Possible sources of antibody for SARS-CoV-2 are human convalescent sera from individuals who have recovered from COVID-19, mAbs, or preparations generated in certain animal hosts, such as genetically engineered cows that produce human antibody (4). Although many types of preparations are or will soon be under development, the only antibody type that is currently available for immediate use is that found in human convalescent sera (Figure 1). As more individuals contract COVID-19 and recover, the number of potential donors will continue to increase.

A general principle of passive antibody therapy is that it is more effective when used for prophylaxis than for treatment of disease. When used for therapy, antibody is most effective when administered shortly after the onset of symptoms. The reason for temporal variation in efficacy is not well understood but could reflect that passive antibody works by neutralizing the initial inoculum, which is likely to be much smaller than that of established disease (5). Another explanation is that antibody works by modifying the inflammatory response, which is also more easily achieved during the initial immune response, a stage that may be asymptomatic (6). As an example, passive antibody therapy for pneumococcal pneumonia was most effective when administered shortly after the onset of symptoms, and there was no benefit if antibody administration was delayed past the third day of disease (7).

For passive antibody therapy to be effective, a sufficient amount of antibody must be administered. When given to a susceptible person, this antibody will circulate in the blood, reach tissues,

Conflict of interest: The authors have declared that no conflict of interest exists.

and provide protection against infection. Depending on the antibody amount and composition, the protection conferred by the transferred immunoglobulin can last from weeks to months.

\section{Historical precedents}

In the early twentieth century convalescent sera was used to stem outbreaks of viral diseases such as poliomyelitis (8), measles $(9,10)$, mumps (11), and influenza (12). A retrospective meta-analysis of eight studies on the use of convalescent sera involving 1703 patients during the $1918 \mathrm{H} 1 \mathrm{~N} 1$ influenza virus pandemic suggested that those who received serum had lower mortality (13). Although the efficacy of convalescent sera varied with the virus and the study, there was consensus at the time that this intervention was useful, and it was used in numerous outbreaks. It is noteworthy that historically, convalescent sera were developed and used in many cases without the means to measure antibody titers or knowledge about viral serotypes, and in clinical studies that did not meet modern criteria for randomization or blinding.

More recently, convalescent serum was used during viral epidemics. In the 2009-2010 H1N1 influenza virus pandemic, convalescent serum antibody preparations obtained by apheresis were used to treat individuals with severe H1N1 2009 infection requiring intensive care (14). Serum-treated individuals manifested reduced respiratory viral burden, serum cytokine responses, and mortality (14). Convalescent serum was also used in the 2013 West African Ebola epidemic. A small nonrandomized study in Sierra Leone revealed significantly longer survival for those treated with convalescent whole blood relative to those who received standard treatment (15). Two patients transferred to the United States and treated with a combination of convalescent serum and an experimental drug also survived (16). There is anecdotal evidence from the H5N1 $(17,18)$ and H7N9 (19) avian flu out- 

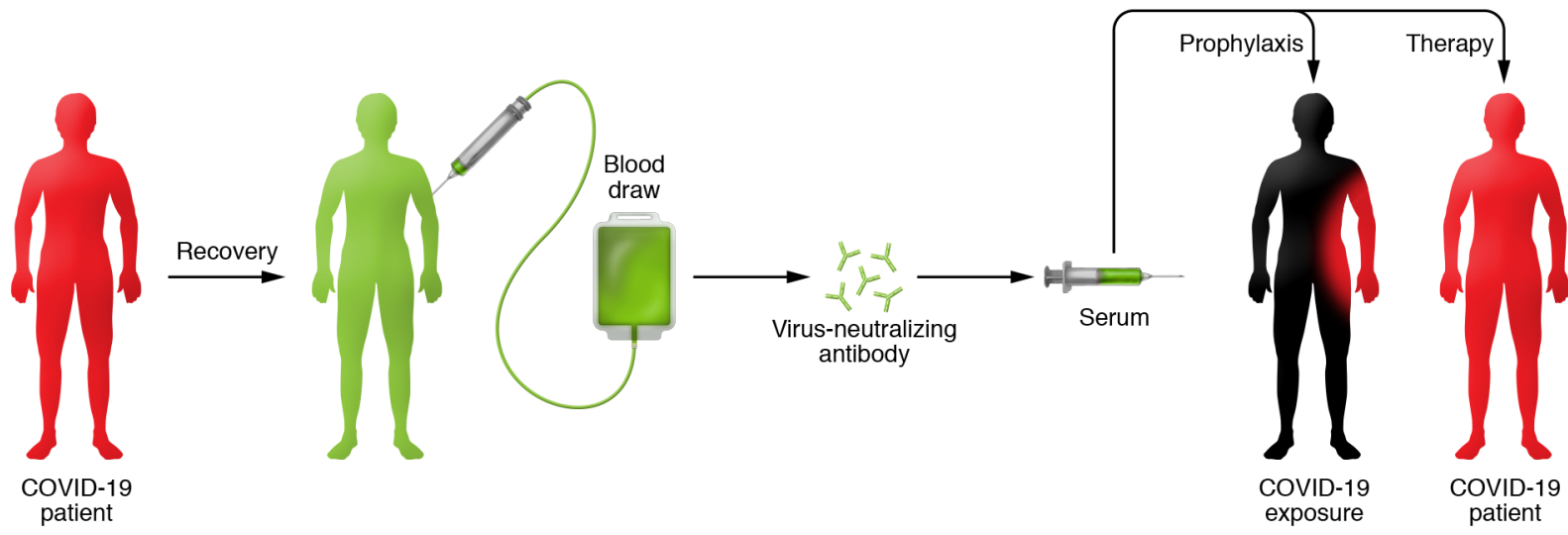

Figure 1. Schematic of the use of convalescent sera for COVID-19. An individual who is sick with COVID-19 and recovers has blood drawn and screened for virus-neutralizing antibodies. Following identification of those with high titers of neutralizing antibody, serum containing these virus-neutralizing antibodies can be administered in a prophylactic manner to prevent infection in high-risk cases, such as vulnerable individuals with underlying medical conditions, health care providers, and individuals with exposure to confirmed cases of COVID-19. Additionally, convalescent serum could potentially be used in individuals with clinical disease to reduce symptoms and mortality. The efficacy of these approaches is not known, but historical experience suggests that convalescent sera may be more effective in preventing disease than in the treatment of established disease.

breaks that use of convalescent sera was effective, with all patients surviving.

Although every viral disease and epidemic is different, these experiences provide important historical precedents that are both reassuring and useful as humanity now confronts the COVID-19 epidemic.

\section{Experience with the use of convalescent sera against coronavirus diseases}

In the twenty-first century, there have been two other epidemics with coronaviruses that were associated with high mortality, SARS1 in 2003 and Middle East respiratory syndrome (MERS) in 2012. The SARS1 epidemic was contained, but MERS became endemic in the Middle East and triggered a secondary major outbreak in South Korea. In both outbreaks, the high mortality and absence of effective therapies led to the use of convalescent serum. The largest study involved the treatment of 80 patients with SARS in Hong Kong (20). Patients treated before day 14 had improved prognosis defined by discharge from hospital before day 22, consistent with the notion that earlier administration is more likely to be effective (20). In addition, those who were PCR positive and seronegative for coronavirus at the time of therapy had improved prognosis (20). There is also some anecdotal information on the use of convalescent serum in seriously ill individuals. Three patients with SARS in Taiwan were treated with
$500 \mathrm{~mL}$ convalescent serum, resulting in a reduction in serum virus titer, and each survived (21). Three patients with MERS in South Korea were treated with convalescent serum, but only two of the recipients had neutralizing antibody in their serum (22). The latter study highlights a challenge in using convalescent sera, namely, that some who recover from viral disease may not have high titers of neutralizing antibody (23). Consistent with this point, an analysis of 99 samples of convalescent sera from patients with SARS showed that 87 had neutralizing antibody, with a geometric mean titer of 1:61 (3). This suggests that antibody declines with time and/or that few patients make high-titer responses. It is also possible that non-neutralizing antibodies are produced that contribute to protection and recovery, as described for other viral diseases (24-26). There are reports that convalescent serum was used for therapy of patients with COVID-19 in China during the current outbreak (27). Although few details are available from the epidemic in China and published studies involved small numbers of patients, the available information suggests that convalescent serum administration reduced viral load and was safe.

\section{Risks and benefits}

COVID-19 convalescent sera can be used for either prophylaxis of infection or treatment of disease. In a prophylactic mode, the benefit of convalescent serum admin- istration is that it can prevent infection and subsequent disease in those who are at high risk for disease, such as vulnerable individuals with underlying medical conditions, health care providers, and those with exposure to confirmed cases of COVID-19. Passive antibody administration to prevent disease is already used in clinical practice. For example, patients exposed to hepatitis $B$ and rabies viruses are treated with hepatitis B immune globulin (HBIG) and human rabies immune globulin (HRIG), respectively. In addition, passive antibody is used for the prevention of severe respiratory syncytial virus (RSV) disease in high-risk infants. Until recently, a polyclonal hyperimmune globulin (RSV-IG) prepared from samples of donors with high serum titers of RSV neutralizing antibody was used, but these preparations have now been replaced by palivizumab, a humanized murine mAb. Used therapeutically, convalescent serum would be administered to those with clinical disease in an effort to reduce their symptoms and mortality. The efficacy of these approaches cannot be inferred without carrying out a controlled clinical trial. Based on the historical experience with antibody administration, it can be anticipated that antibody administration would be more effective in preventing disease than in the treatment of established disease (12).

Risks of passive administration of convalescent sera fall into two categories, known and theoretical. Known risks are 
those associated with transfer of blood substances, which include inadvertent infection with another infectious disease agent and reactions to serum constituents, including immunological reactions such as serum sickness. With modern blood banking techniques that screen for blood-borne pathogens and match the blood type of donors and recipients, the risks of inadvertently transferring known infectious agents or triggering transfusion reactions are low. However, convalescent sera used in a therapeutic mode would likely be administered to individuals with pulmonary disease, in whom plasma infusion carries some risk for transfusionrelated acute lung injury (TRALI) (28), and this should be a consideration in the risk-benefit assessment. The theoretical risk involves the phenomenon of antibodydependent enhancement of infection (ADE). ADE can occur in several viral diseases and involves an enhancement of disease in the presence of certain antibodies. For coronaviruses, several mechanisms for ADE have been described, and there is the theoretical concern that antibodies to one type of coronavirus could enhance infection to another viral strain (29). It may be possible to predict the risk of $\mathrm{ADE}$ of SARS-CoV-2 experimentally, as proposed for MERS (29). Since the proposed use of convalescent sera in the COVID-19 epidemic would rely on preparations with high titers of neutralizing antibody against the same virus, SARS2-CoV-2, ADE may be unlikely. The available evidence from the use of convalescent sera in patients with SARS1 and MERS (30), and anecdotal evidence from its use in 245 patients with COVID-19 (27), suggest it is safe. Nevertheless, in convalescent serum trials, caution and vigilance to identify any evidence of enhanced infection will be required.

Another theoretical risk is that antibody administration to those exposed to SARS-CoV-2 may prevent disease in a manner that attenuates the immune response, leaving such individuals vulnerable to subsequent reinfection. In this regard, passive antibody administration before vaccination with respiratory syncytial virus was reported to attenuate humoral but not cellular immunity (31). This concern could be investigated as part of a clinical trial by measuring immune responses in those exposed and treated with convalescent sera to prevent disease. If the risk proved real, these individuals could be vaccinated against COVID-19 when a vaccine becomes available.

Given that historical and current anecdotal data on use of convalescent serum suggest it is safe in coronavirus infection, the high mortality of COVID-19, particularly in elderly and vulnerable persons, suggests that the benefits of its use in those at high risk for or with early disease outweigh the risks. However, for all cases where convalescent serum administration is considered, a risk-benefit assessment must be conducted to assess individual variables. These considerations were invoked recently with the decision to use mAbs in the treatment of Ebola virus disease (32).

\section{Deployment and proposed use}

To deploy convalescent serum administration for COVID-19 the following six conditions must be met: (i) availability of a population of donors who have recovered from the disease and can donate convalescent serum; (ii) blood banking facilities to process the serum donations; (iii) availability of assays, including serological assays, to detect SARS-CoV-2 in serum and virological assays to measure viral neutralization; (iv) virology laboratory support to perform these assays; (v) prophylaxis and therapeutic protocols, which should ideally include randomized clinical trials to assess the efficacy of any intervention and measure immune responses; and (vi) regulatory compliance, including institutional review board approval, which may vary depending on location. Ideally, the use of convalescent serum would involve multiple centers, follow randomized control protocols, and have a single center as a governing body. Each of these conditions should be available in developed areas affected by COVID-19. At least one pharmaceutical company, Takeda, is gearing up to generate antibody preparations against SARS2CoV-2 from COVID-19 convalescent sera (33). Producing highly purified preparations containing a high titer of neutralizing antibodies against SARS2-CoV-2 is preferable to convalescent sera given that these are safer and have higher activity. Unfortunately, such preparations will not be available for many months, whereas locally produced convalescent sera could be available much sooner.
We anticipate that once the necessary regulatory permissions are in place, individuals who recover from COVID-19 can be approached to donate blood for serum preparation or antibody isolation through apheresis. Recovery from COVID-19 will be assessed clinically, and such individuals must be shown to free of SARS-CoV-2, including in their blood by appropriate viral nucleic acid screening. Donated blood products will be screened for infectious agents according to current blood banking practices, and individual sera will be studied for specific antibody content and neutralizing activity to SARS-CoV-2. Depending on the volumes needed and the neutralizing activity of donated convalescent sera, these could be pooled or used individually, and preparations for clinical use would be treated for pathogen attenuation. At this time, we do not know what an effective neutralizing titer would be in a susceptible individual given passive antibody therapy for prophylaxis, and determining this parameter would be part of the study design. Similarly, we do not know what doses would be effective therapeutically. We do know that when convalescent serum was used to prevent measles or mumps the amounts used were in the order of $10-40 \mathrm{cc}(10,11)$. In contrast, when convalescent serum was used to treat severe disease in soldiers with 1918 influenza, the amounts given were in the hundreds of milliliters (34). These older studies claimed efficacy even though convalescent serum was given without any knowledge of neutralizing titers. Those experiences suggest that even small amounts of antibody may prevent and/or treat infection. Hence, we can anticipate that effective prophylactic doses would be much smaller than therapeutic doses. This makes sense, since the infecting inoculum is likely to be much smaller than the viral burden during severe disease.

COVID-19 convalescent sera could be used to treat individuals with early symptoms and prevent disease in those exposed. Today, nurses, physicians, and first responders exposed to known cases of COVID-19, some of whom have developed disease, are being quarantined, which threatens to collapse the health care system. It is anticipated that convalescent serum will prevent SARS-CoV-2 infection in those to whom it is administered. If this is established, indi- 
viduals who receive convalescent sera may be able to avoid a period of quarantine. This could allow them to continue their critical function as health care providers. Convalescent sera could also be used to prevent disease among family members caring for COVID-19 patients at home. Clearly, the use of convalescent serum would be a stopgap measure that could be used in the midst of the current epidemic. However, even local deployment will entail considerable coordination between different entities, such as infectious disease specialists, hematologists, blood banking specialists, and hospital administrators. Hence, as we are in the midst of a worldwide pandemic, we recommend that institutions consider the emergency use of convalescent sera and begin preparations as soon as possible. Time is of the essence.

\section{Acknowledgments}

We are grateful to Shmuel Sholam for helpful comments and suggestions. AC was supported in part by NIH grants HL059842, AI052733 and AI152078. LP was supported in part by NIH grants 6R01AG045044-07, AI123654, and AI143453.

Address correspondence to: Arturo Casadevall, 615 N. Wolfe Street, Room E5132, Baltimore, Maryland 21205, USA. Phone: 410.955.3457; Email: acasadevall@jhu.edu.

1. Casadevall A, Scharff MD. Return to the past: the case for antibody-based therapies in infectious diseases. Clin Infect Dis. 1995;21(1):150-161.

2. Casadevall A, Dadachova E, Pirofski LA. Passive antibody therapy for infectious diseases. Nat Rev Microbiol. 2004;2(9):695-703.

3. Zhang JS, et al. A serological survey on neutralizing antibody titer of SARS convalescent sera. JMed Virol. 2005;77(2):147-150.

4. Beigel JH, et al. Safety and tolerability of a novel, polyclonal human anti-MERS coronavirus antibody produced from transchromosomic cattle: a phase 1 randomised, double-blind, single-dose-escalation study. Lancet Infect Dis. 2018;18(4):410-418.

5. Robbins JB, Schneerson R, Szu SC. Perspective: hypothesis: serum IgG antibody is sufficient to confer protection against infectious diseas- es by inactivating the inoculum. J Infect Dis. 1995;171(6):1387-1398.

6. Casadevall A, Pirofski LA. Antibody-mediated regulation of cellular immunity and the inflammatory response. Trends Immunol. 2003;24(9):474-478.

7. Casadevall A, Scharff MD. Serum therapy revisted: animal models of infection and development of passive antibody therapy. Antimicrob Agents Chemother. 1994; 38(8):1695-1702.

8. Park WH. Therapeutic use of antipoliomyelitits serum in preparalytic cases of poliomyelitis. JAMA. 1932;99:1050-1053.

9. Park WH, Freeman RG. The prophylactic use of measles convalescent serum. JAMA. 1926;87(8):556-558.

10. Gallagher JR. Use of convalescent measles serum to control measles in a preparatory school. Am J Public Health Nations Health. 1935;25(5):595-598.

11. Rambar AC. Mumps; use of convalescent serum in the treatment and prophylaxis of orchitis. Am J Dis Child. 1946;71:1-13.

12. Luke TC, Casadevall A, Watowich SJ, Hoffman SL, Beigel JH, Burgess TH. Hark back: passive immunotherapy for influenza and other serious infections. Crit Care Med. 2010;38(4 suppl):e66-e73

13. Luke TC, Kilbane EM, Jackson JL, Hoffman SL. Meta-analysis: convalescent blood products for Spanish influenza pneumonia: a future $\mathrm{H} 5 \mathrm{~N} 1$ treatment? Ann Intern Med. 2006;145(8):599-609.

14. Hung IF, et al. Convalescent plasma treatmen reduced mortality in patients with severe pandemic influenza A (H1N1) 2009 virus infection. Clin Infect Dis. 2011;52(4):447-456.

15. Sahr F, et al. Evaluation of convalescent whole blood for treating Ebola virus disease in Freetown, Sierra Leone. J Infect. 2017;74(3):302-309.

16. Kraft CS, et al. The Use of TKM-100802 and convalescent plasma in 2 patients with Ebola virus disease in the United States. Clin Infect Dis. 2015;61(4):496-502.

17. Kong LK, Zhou BP. Successful treatment of avian influenza with convalescent plasma. Hong Kong Med J. 2006;12(6):489.

18. Zhou B, Zhong N, Guan Y. Treatment with convalescent plasma for influenza A (H5N1) infection. N Engl J Med. 2007;357(14):1450-1451.

19. Wu XX, Gao HN, Wu HB, Peng XM, Ou HL, Li LJ. Successful treatment of avian-origin influenza A (H7N9) infection using convalescent plasma. Int J Infect Dis. 2015;41:3-5.

20. Cheng Y, et al. Use of convalescent plasma therapy in SARS patients in Hong Kong. Eur J Clin Microbiol Infect Dis. 2005;24(1):44-46.

21. Yeh KM, et al. Experience of using convalescent plasma for severe acute respiratory syndrome among healthcare workers in a Taiwan hospital.
JAntimicrob Chemother. 2005;56(5):919-922.

22. $\mathrm{Ko} \mathrm{JH}$, et al. Challenges of convalescent plasma infusion therapy in Middle East respiratory coronavirus infection: a single centre experience. Antivir Ther (Lond). 2018;23(7):617-622.

23. Arabi YM, et al. Feasibility of using convalescent plasma immunotherapy for MERS-CoV infection, Saudi Arabia. Emerging Infect Dis. 2016;22(9):1554-1561.

24. van Erp EA, Luytjes W, Ferwerda G, van Kasteren PB. Fc-mediated antibody effector functions during respiratory syncytial virus infection and disease. Front Immunol. 2019;10:548.

25. Jenks JA, Goodwin ML, Permar SR. The roles of host and viral antibody fc receptors in herpes simplex virus (HSV) and human cytomegalovirus (HCMV) infections and immunity. Front Immunol. 2019;10:2110.

26. Gunn BM, et al. A role for Fc function in therapeutic monoclonal antibody-mediated protection against ebola virus. Cell Host Microbe. 2018;24(2):221-233.e5

27. China puts 245 COVID-19 patients on convalescent plasma therapy. News release. Xinhua. February 28, 2020. Accessed March 10, 2020. http://www.xinhuanet.com/english/202002/28/c_138828177.htm

28. Gajic O, et al. Transfusion-related acute lung injury in the critically ill: prospective nested case-control study. Am J Respir Crit Care Med. 2007;176(9):886-891.

29. Wan Y, et al. Molecular mechanism for antibody-dependent enhancement of coronavirus entry. J Virol. 2020;94(5):e02015-19.

30. Mair-Jenkins J, et al. The effectiveness of convalescent plasma and hyperimmune immunoglobulin for the treatment of severe acute respiratory infections of viral etiology: a systematic review and exploratory meta-analysis. J Infect Dis. 2015;211(1):80-90.

31. Crowe JE, Firestone CY, Murphy BR. Passively acquired antibodies suppress humoral but not cell-mediated immunity in mice immunized with live attenuated respiratory syncytial virus vaccines. JImmunol. 2001;167(7):3910-3918.

32. Mulangu S, et al. A randomized, controlled trial of Ebola virus disease therapeutics. $N$ Engl J Med. 2019;381(24):2293-2303.

33. Hopkins JS. Drugmaker Takeda Is Working on Coronavirus Drug. Wall Street Journal. https://www.wsj.com/articles/drugmakertakeda-is-working-on-coronavirusdrug-11583301660. Published March 4, 2020. Accessed March 10, 2020.

34. McGuire LW, Redden WR. The use of convalescent human serum in influenza pneumoniaa preliminary report. Am J Public Health (N Y). 1918;8(10):741-744. 